\title{
Carbon monoxide: a rare cause of myocardial ischemia
}

\author{
Kanaan Mansoor, MD', Greg Parkins, DO', Laurie Wilson, MD', \\ Jerry White, DO' ${ }^{1}$, Brandon S. Shiflett, MD' ${ }^{1}$, Aman Ajmeri, MD', \\ Fuad Zeid, MD'
}

ABSTRACT

In an acute care setting, chemical asphyxiants (CA) are culprits for debilitating injury. Carbon monoxide (CO) is one well known CA which causes hypoxic injury to cardiovascular and neurological tissue. $\mathrm{CO}$ poisoning is one of the leading causes of death in USA. As many as $6 \%$ of patients who get admitted with CO poisoning in the USA have acute myocardial infarction. A strong positive correlation of $\mathrm{CO} \mathrm{Hb}$ concentration has been established with increased incidence of myocardial infarctions (MI). We present a case of a 75-year-old male with complaints of chest discomfort, dyspnea, diaphoresis that was attributed to CO poisoning. Over the course of his stay he had two sets of positive serial troponins and was diagnosed with a non-ST elevation MI. Most of the recent literature focuses on ST elevation and T wave inversions in patients with CO poisoning. Contrary to this, our patient did not exhibit any EKG changes at any point during his hospital course. $\mathrm{CO}$ poisoning can cause fatal complications including an MI.

\section{KEYWORDS}

Carbon Monoxide, CO, MI, Myocardial Infarction, Myocardial Ischemia
Author affiliations are listed at the end of this article.

Correspondence to: Kanaan Mansoor, MD Marshall University Joan C. Edwards School of Medicine kanaanm@gmail.com

\section{BACKGROUND}

In an acute care setting, chemical asphyxiants (CA) are culprits for debilitating injury. Carbon monoxide (CO) is one well known CA which causes hypoxic injury to cardiovascular and neurological tissue. ${ }^{1}$ $\mathrm{CO}$ is a colorless, non-irritating, odorless gas with a density of 0.97 , which has the potential to go undetected and cause serious complications. ${ }^{2} \mathrm{CO}$ causes injury because it has 60 times higher affinity to bind with oxygen carrying proteins (hemoglobin and myoglobin), leading to the formation of carboxyhemoglobin $(\mathrm{COHb})$, thereby reducing the oxygen available to the mitochondria and leading to impaired oxidative phosphorylation. ${ }^{3}$ An additive pathway for $\mathrm{CO}$ to injure cells is by direct toxic injury to mitochondria at the level of cytochrome c oxidase. ${ }^{2}$ A strong positive correlation of $\mathrm{COHb}$ concentration has been established with increased incidence of myocardial infarctions (MI). ${ }^{4}$ We present a case of a 75-year-old male with incidental CO poisoning who sustained a non-ST elevation myocardial infarction (NSTEMI).

\section{CASE}

A 75-year-old Caucasian male with a past medical history significant for hypertension and a 75 pack year history of smoking presented to the emergency department at 2030 via emergency medical services (EMS) after complaining of dyspnea, dizziness, nausea, diaphoresis, and chest discomfort. He reported that he was working on his automobile in an unventilated garage with the doors closed. Incidentally, his engine was running for approximately 10 minutes until he noted the aforementioned symptoms. He immediately left the garage and called the EMS. He described the chest pain as pressure like which spread across his 
chest. When EMS arrived he was given supplemental oxygen; this helped to quell his symptoms which had largely resolved by the time he arrived at the ED.

On arrival his blood pressure was $162 / 91 \mathrm{mmHg}$, his heart rate was $97 \mathrm{bpm}$, and his respiratory rate was $20 \mathrm{bpm}$ with an oxygen saturation of $97 \%$. He was provided $100 \%$ supplemental oxygen via a non-rebreather mask. His laboratory workup was significant for CO level of $19.9 \%$, a serum troponin I of $1.146 \mathrm{ng} / \mathrm{dL}$ and a serum creatinine of $1.45 \mathrm{mg} / \mathrm{dL}$. His ABG demonstrated a pH of 7.45, a pCO2 of $38 \mathrm{mmHg}$, and a pO2 of $130 \mathrm{mmHg}$. His EKG did not suggest any acute changes except for a potential prior inferior wall MI (Figure 1). He was admitted to the ICU at 2150 and the cardiology team was consulted. Oxygen saturation measured by pulse oximetry consistently yielded $100 \%$. Repeat serum troponin I levels were collected at $\mathrm{T}+2$ hour and $\mathrm{T}+4$ hour intervals resulting in significant elevations, to $10.6 \mathrm{ng} / \mathrm{mL}$ and $12.7 \mathrm{ng} / \mathrm{mL}$ respectively. The diagnosis of Type 2 NSTEMI was applied, and a weight-based heparin infusion was started.

His CO was assessed after every three hours, which showed progressive improvement to levels of $7.7 \%$ at 0016 and $4.7 \%$ at 0336 hours. At approximately 0500 hours he complained of mild chest discomfort. Following examination, a repeat EKG was ordered, and serum troponin I levels again trended, resulting in $20.6 \mathrm{ng} / \mathrm{mL}, 21.4 \mathrm{ng} / \mathrm{mL}$, and $20.4 \mathrm{ng} / \mathrm{mL}$ at $\mathrm{T}+0$, $\mathrm{T}+2$, and $\mathrm{T}+4$ hours respectively.

The patient's CO levels improved and reached $3.7 \%$ at 0632 . The patient continued to receive supplemental oxygen via a $100 \%$ non-rebreather

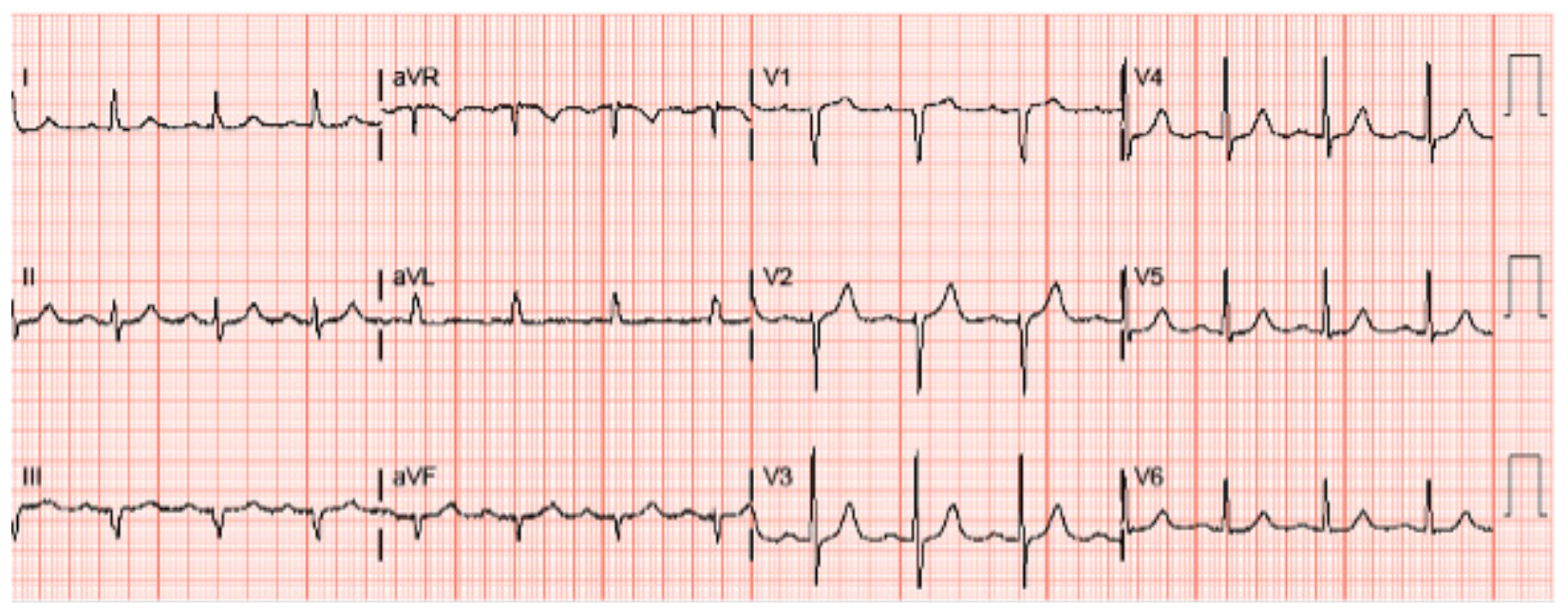

FIGURE 1 EKG on presentation, showing nonspecific changes

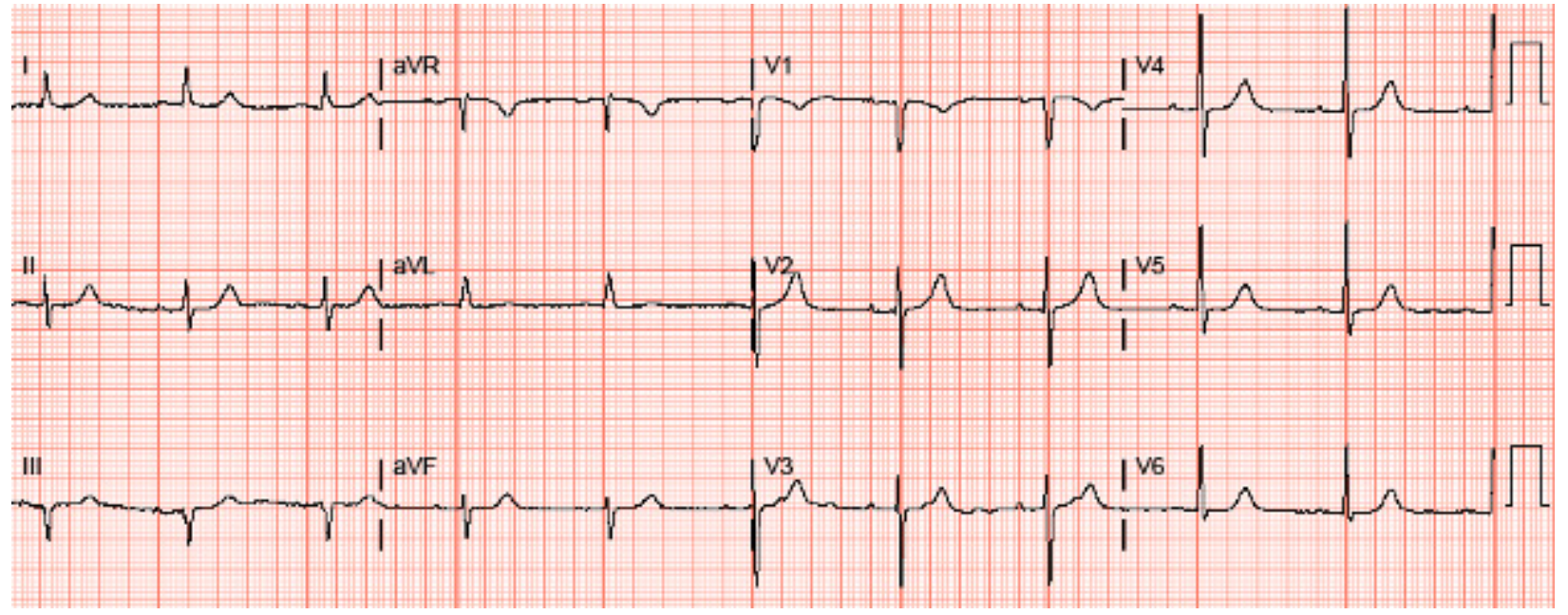

FIGURE 2 EKG on discharge 
mask. Upon further inquiry, the patient revealed an undocumented history of $\mathrm{MI}$ in the past. An echocardiogram was obtained and demonstrated an ejection fraction (EF) of $>55 \%$ and a right ventricular systolic pressure (RVSP) of $26.8 \mathrm{mmHg}$ with no apparent wall motion defect. He was continued on a heparin infusion and remained symptom free. A nuclear myocardial perfusion scan was performed and suggested no evidence of myocardial infarction or ischemia, with an EF of 54\%. The patient's CO levels reached $1.2 \%$ just prior to discharge. EKG done prior to discharge did not show any changes (Figure 2).

\section{DISCUSSION}

$\mathrm{CO}$ and its association with myocardial ischemia and infarction has been studied and reported previously. Mujib et al reported that $6 \%$ of patients had acute myocardial infarctions after $\mathrm{CO}$ poisoning, and out of those $9 \%$ had in-hospital mortality. ${ }^{5}$ The most plausible hypothesis suggests that $\mathrm{COHb}$ diminishes oxygen transportation, leading to myocardial tissue hypoxia. ${ }^{1,2,4}$ Other studies have also proposed that CO induces vasospasm, reduces blood flow, and increases permeability of the coronary vasculature, thereby promoting platelet aggregation and thrombus formation. ${ }^{2}$ Kaya et al discussed that $\mathrm{CO}$ inhibits mitochondrial cytochrome c oxidase which reduces the level of glutathione, which in turn causes hypoxia, lactic acidosis, and eventually apoptosis in cells. ${ }^{4}$

Satran et al studied moderate to severe CO poisoning and serum troponin levels in 230 patients and found that $35 \%$ of the patients with CO poisoning have elevated serum troponin levels. ${ }^{6}$ The trend of elevation in serum troponin levels in our patient was similar to the trend previously reported, which spans from 2-8 hours. ${ }^{1,7,8}$ The rise in troponin levels correlates to the half-life of $\mathrm{CO}$ in patients. It has been reported that $\mathrm{CO}$ has a half-life of 300 minutes if the patient is breathing room air, though this is decreased to 90 minutes with use of a non-breather mask and 30 minutes if hyperbaric oxygen treatment is provided. ${ }^{9}$ Considering the time needed for the serum troponin level to rise after myocardial injury and the half-life of $\mathrm{CO}$, it could be hypothesized that a patient should demonstrate a rise in serum troponin levels in approximately 2-8-hours after exposure.

Most of the recent literature focuses on ST elevation and T wave inversions in patients with CO poisoning. ${ }^{7,8,9,10}$ Contrary to this, our patient did not exhibit any EKG changes at any point during his hospital course. It is reported that other cardiac groups may not show any ischemic changes on EKG. Rab et al reported that in patients with a cardiac arrest and no STEMI on EKG, approximately 33\% have a culprit vessel and $70 \%$ of those are occluded. ${ }^{11}$ Rasoul et al found that non-diagnostic EKGs (no ST elevation) were present in $18 \%$ of patients with a left circumflex related infarct and in $7 \%$ of the right coronary artery related infarcts. ${ }^{12}$ This shows that a non-ischemic EKG does not automatically exclude on going ischemia.

As concluded by Buckley et al in a recent Cochrane review, supplemental $100 \%$ oxygen delivery via a non-rebreather mask remains the mainstay of treatment in CO poisoning. ${ }^{13}$ Patients with ST elevation should receive coronary angiography, as $\mathrm{CO}$ poisoning is known to exacerbate underlying coronary arteriosclerosis. ${ }^{1,9}$ While hospitalized with a non-ST elevation MI, an echocardiogram and nuclear stress test should be considered, as these modalities would be more cost effective and predictive of cardiac function. Our patient did not demonstrate any apparent wall motion abnormalities and had an EF of $>55 \%$, which did not warrant additional intervention.

\section{CONCLUSION}

CO poisoning can cause fatal complications for patients, hence diagnostic vigilance is required for physicians who encounter such patients, especially during winter months. Modalities such as EKG, echocardiogram, and serum cardiac markers should be utilized to assess cardiac function. Coronary angiography should be performed in accordance with the guidelines of American College of Cardiology. Utilization of hyperbaric oxygen therapy should be considered, but more research is required to establish use of hyperbaric oxygen treatment in 
patients with $\mathrm{CO}$ poisoning and myocardial ischemia.

\section{AUTHOR AFFILIATIONS}

1. Marshall Uniersity Joan C. Edwards School of Medicine, Huntington, West Virginia

\section{REFERENCES}

1. Kim S, Lim JH, Kim Y, Oh S, Choi WG. A case of acute carbon monoxide poisoning resulting in an ST elevation myocardial infarction. Korean Circ J. 2012;42(2):133-5.

2. Türkdogan KA, Karabacak M, Kapci M, Akpinar O. Rare cause of acute myocardial infarction: carbon monoxide poisoning. Acta Medica Mediterr. 2014;30:743.

3. Dragelytė G, Plenta J, Chmieliauskas S, Jasulaitis A, Raudys R, Jovaiša T, et al. Myocardial Rupture following carbon monoxide poisoning. Case Reports Crit Care. 2014;2014:281701.

4. Kaya H, Coşkun A, Beton O, Zorlu A, Kurt R, Yucel $\mathrm{H}$, et al. $\mathrm{COHgb}$ levels predict the long-term development of acute myocardial infarction in CO poisoning. Am J Emerg Med. 2016;34(5):8404.

5. Mujib MU, Khera S, Kolte D, Palaniswamy C, Garg $\mathrm{J}$, Aronow WS. Acute myocardial infarction and in-hospital mortality after carbon monoxide poisoning: an analysis of the nationwide inpatient sample 2002-2010. J Am Coll Cardiol. 2013;61(10):E55.

6. Satran D, Henry CR, Adkinson C, Nicholson $\mathrm{Cl}$, Bracha Y, Henry TD. Cardiovascular manifestations of moderate to severe carbon monoxide poisoning. J Am Coll Cardiol. 2005;45(9):1513-6.

7. Negatu Y, Healy W, Taylor M, Stasek J. Acute ST elevation myocardial infarction related to carbon monoxide poisoning in a young patient without coronary artery disease. Chest. 2016;150(4):262A.

8. Ebisuno $S$, Yasuno M, Yamada Y, Nishino $Y$, Hori $M$, Inoue $M$, et al. Myocardial infarction after carbon monoxide poisoning. Kardiol Pol. 1986;67(10):1107-9.

9. Hsu P-CC, Lin T-HH, Su H-MM, Lee H-CC, Huang $\mathrm{C}-\mathrm{HH}$, et al. Acute carbon monoxide poisoning resulting in st elevation myocardial infarction: a rare case report. Kaohsiung J Med Sci. 2010;26(5):271-5.

10. Robert A, Weaver LK. C45: Carbon monoxide (CO) poisoning precipitation of NSTEMI - A Case Report - Hyperbaric Medical Society [Internet]. Intermountain Healthcare Research. 2015 [cited 2018 Feb 14]. p. C45. Available from: https://www.uhms.org/2015-annualscientific-meeting-presentations/friday-june-19/ session-c-clinical-hbo2-therapy-1/session-cposters/c45-carbon-monoxide-co-poisoningprecipitation-of-nstemi-a-case-report.html

11. Rab T, Kern KB, Tamis-Holland JE, et al. Cardiac arrest: a treatment algorithm for emergent invasive cardiac procedures in the resuscitated comatose patient. J Am Coll Cardiol. 2015;66:6273.

12. Rasoul $S$, de Boer MJ, Suryapranata $H$, et al. Circumflex artery-related acute myocardial infarction: limited ECG abnormalities but poor outcome. Neth Heart J. 2007;15(9):286-90.

13. Buckley NA, Juurlink DN, Isbister G, Bennett $\mathrm{MH}$, Lavonas EJ. Hyperbaric oxygen for carbon monoxide poisoning. Cochrane Database Syst Rev. 2011;(4):CD002041. 